\title{
Reaching Men at Highest Risk for Undetected Prostate Cancer
}

\author{
Rita E. Arras-Boyd Roger E. Boyd Kay Gaehle \\ Southern Illinois University Edwardsville
}

\begin{abstract}
Annual screening for prostate cancer, although controversial, should be offered to men over age 45 . Community-based prostate cancer education and screening offer a way to reach those at highest risk for undetected prostate cancer, AfricanAmerican men and men without healthcare providers. This study used logistic regression to predict attendance at community-based education and screening events. Being white, not having a healthcare provider, desire for information, and knowledge of prostate cancer status all independently predicted attendance. While these community education and screening events attracted men without healthcare providers, they were not as effective in attracting African-American men. Findings have implications for planning community education and screening programs that target high risk men.
\end{abstract}

Keywords: prostate cancer, prostate cancer screening, African-American men, community health education, risk factors

African-Americans are more likely to get cancer, be diagnosed later, and subsequently die from cancer than other Americans (American Cancer Society, 2008). Prostate cancer (PC) is the most commonly occurring cancer and the second leading cause of cancer deaths in African-American men. The reasons for PC healthcare disparities are complex and include genetics, lifestyle and environment, as well as access to treatment, education, and screening. There is also controversy around the risk-benefit balance of screening - which men should be screened, the most appropriate screening mechanism, and interpretation of results. Until these issues are resolved, the most prudent, ethical approach is to provide education so men can make an informed decision on whether or not to be screened.

A community-based, education and screening program targeted men at highest risk for undetected PC, African-Americans and those with healthcare access barriers. The program was implemented in a Southwestern Illinois county populated by 250,000 people, among whom one out of three residents are African-American (U.S. Department

Rita E. Arras-Boyd, Roger E. Boyd and Kay Gaehle, Southern Illinois University Edwardsville.

Correspondence concerning this article should be addressed to Rita E. Arras-Boyd, Southern Illinois University Edwardsville School of Nursing, Department of Family Health and Community Health Nursing, Box 1066 Edwardsville, IL 62026-1066. Email: rarras@siue.edu

International Journal of Men's Health, Vol. 8, No. 2, Summer 2009, 116-128. (C) 2009 by the Men's Studies Press, LLC. http://www.mensstudies.com. All rights reserved. jmh.0802.116/\$14.00 • DOI: 10.3149/jmh.0802.116•Url: http://dx.doi.org/10.3149/jmh.0802.116

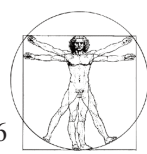


of Commerce, Census Bureau, 2005). Prostate cancer incidence rates and death rates among African-American and white men in the county parallel national trends (Illinois Department of Public Health, 2008). A state public health grant provided funding for free PC education and the offer of prostate specific antigen (PSA) screening. Education and screening sessions were held in 19 locations throughout the county.

Pre-intervention personal and family health histories, attitudes towards screening, and access to care, along with a pretest measuring knowledge of PC screening benefits and risks were offered to all men attending education and screening events. Education was then provided by a health educator for all attendees which consisted of a 10-minute oral presentation and a question and answer session, supplemental by written brochures. The goal of the educational component was to provide attendees the basis for making an informed decision about whether or not to participate in PC screening. Table 1 displays the content and concepts addressed in the educational component.

Table 1

Content Presented at Education and screening Events

\begin{tabular}{lllll}
\hline Concept & Content & \multicolumn{2}{c}{ Delivery } \\
\cline { 2 - 5 } Method & & \multicolumn{2}{c}{ Slide/Script/Booklet } \\
\hline Prostate / Prostate Cancer & & & \\
& Definition; Anatomy, Role of Prostate; & X & X & X \\
& Age, Race, Family History \& Lifetime Risk & X & X & \\
& Most PC Grows Slowly; Localized vs. Metastasis & & X & X
\end{tabular}

Signs / Symptoms

Common Symptoms; Non-Specific (as in BPH) $\quad$ x $\quad$ x $\quad$ x

Sometimes Asymptomatic

Screening for $\mathrm{PC}^{*}$

PSA, DRE

Screening guidelines \& controversy

Positive screen necessitates follow-up

False positive and some of the reasons

Diagnosing PC

Return of results \& follow-up referrals

$\begin{array}{lll}\text { X } & \text { X } & \text { X } \\ \text { X } & \text { X } & \text { X } \\ \text { X } & \text { X } & \text { X } \\ \text { X } & \text { X } & \text { X } \\ \text { X } & \text { X } & \end{array}$

Biopsy usual diagnostic tool

Risks of Biopsy

X $\quad \mathrm{X} \quad \mathrm{X}$

Treating PC

Many treatment options

$\begin{array}{ccc} & \text { X } & \text { X } \\ \text { X } & \text { X } & \text { X } \\ & \text { X } & \text { X }\end{array}$

*Includes informed decision making elements regarding PC screening controversy. 
A PSA test was provided for men who wished to be screened and a knowledge post-test was offered. The PROCASE was selected as the pretest-posttest knowledge test because it focuses on elements of informed decision making (the possibility of false positive results) (Radosevich et al., 2004). No studies were identified comparing perspectives of attendees to non-attendees; therefore, 87 male residents who did not attend education and screening events were also queried for PC knowledge, attitudes and history. This comparison provides insight into factors that motivate or inhibit men from seeking education and screening for PC.

\section{Review of Related Literature}

In the last decade increasing attention has been directed at eliminating disparities in health and healthcare in the United States (Healthy People, 2000). Men, by virtue of gender, experience significant health disparities. American men have higher death rates in all of the ten leading causes of death and die 5.5 years sooner than women (National Center for Health Statistics, 2004). Contributing behavioral and social factors include the propensity to underrate risk for health problems, perceptions of invulnerability, and inadequate knowledge of diseases (Courtenay, 2000). Cancer awareness follows this pattern. Men underestimate their risk of dying from cancer (McCreary, Gray, \& Grace, 2006; Reagan, 1997).

African-Americans have the highest cancer death rates among racial or ethnic group in the United States (American Cancer Society, 2008). These disparities are greatest for prostate cancer, African-American men being 1.6 times more likely to be diagnosed with and 2.4 times more likely to die from PC than white American men. African-American men are also more likely to be diagnosed with PC when the cancer is in an advanced stage. Death rates for PC among African-American men peaked in the 1990s and have been declining since then, although at a slower rate of decline than for white men. Lack of knowledge, screening, and access to health care all contribute to PC disparities for African-American men (American Cancer Society).

Studies have shown that African-American men are often unaware of their increased risk for prostate cancer. Thirty-six percent of African-American men over age 50 in a telephone survey were unaware or reported they did not believe they were at increased risk of developing the cancer (Steele, Miller, Maylahan, Uhler, \& Baker, 2000). Nivens, Herman, Weinrich and Weinrich (2001) found 38\% of older, African-American men had not heard or read anything about PC during the past 12 months. AfricanAmerican men want accurate information about the cancer and PC screening (Plowden, 2006) and men who have access to PC information are more likely to participate in screening (Nivens et al., 2001).

Despite increased risk, African-American men are less likely than white men to be screened for prostate cancer (Abbott, Taylor, \& Barber, 2001; Agho, \& Lewis, 2001). Besides lack of awareness, there are social-environmental factors associated with PC screening for African-American men. Social support from significant others has been shown to influence screening (Plowden, 2006; Woods, Montgomery, Belliard, Ramírez- 
Johnson, \& Wilson, 2004). Settings where education and screening are offered may influence participation; therefore, many successful events have been situated in community settings such as barbershops (Cowart, Brown, \& Biro, 2004), churches (Weinrich et al., 1998b) and places of employment (Weinrich, Boyd, Bradford, Mossa, \& Weinrich, 1998a). Convenience of location may account for robust participation in community settings, but the influence of leaders in community settings may also effect participation. Awareness of a congregation member diagnosed with prostate cancer predicted screening in one study (Weinrich et al., 1998b), while barbers were seen as gatekeepers in the Cowart et al. (2004) project. Barbers, supervisors, housing directors, and other leaders were described as critical factors in participation in another, multi-setting study (Weinrich et al., 1998a). African-American men seem to prefer community-based activities for PC detection, while white men prefer an individual-oriented approach to PC screening (McFall, Hamm, \& Volk, 2006). Healthcare provider recommendations to participate in PC screening may provide access to screening and also serve as a powerful influence for men to participate in screening (Nivens et al., 2001; Steele et al., 2000).

There is considerable controversy surrounding PC screening, including questions about the risk-benefit balance of screening, which men should be screened, the most appropriate screening mechanism, and interpretation of results (AHRQ, 2006; Brawley, 2001; Burack \& Wood, 1999; Chan et al., 2003; Plowden, 2006). The American Cancer Society (ACS) recommends annual screening with the PSA blood test combined with a digital rectal exam (DRE) for men over 50 who are expected to live 10 additional years. African-American men should be offered screening at age 45 , and men with a first-degree relative with PC offered screening beginning at age 40 (Smith, Cokkinindes, \& Eyre, 2006). However, one study concluded that for African-American men in particular, the DRE may be objectionable and discourage participation in screening (Nagler et al., 2004). For men for whom a DRE is an obstacle to testing, a PSA alone is an acceptable alternative (Smith et al., 2006). Unfortunately, PSA blood tests can also be elevated in non-malignant conditions, necessitating more invasive testing. While the U.S. Preventative Services Task Force states there is insufficient evidence that routine annual PC screening increases length and quality of life, the National Cancer Institute makes recommendations similar to ACS for PC testing while attempting to resolve the controversy through a large, longitudinal study, the Prostate, Lung, Colorectal Screening Trial (National Cancer Institute, 2007). One of the purposes of the present study was to examine understanding of this controversy among at-risk men and its impact on PC screening decision-making.

Woods, Montgomery, Belliard, Ramiriz-Johnson, and Wilson (2004) suggest that recommendations for PC screening were based on an insufficient number of AfricanAmerican men, clouding the picture for this high-risk group. Participation in mass screenings (a scenario frequently conveyed by the media) poses an ethical dilemma in public health (Chan et al., 2003). The dilemma is whether men who are insufficiently informed as to the benefits and risks of PC screening should be subjected to unnecessary biopsies, worry, and overzealous treatment for non-aggressive cancers, thus in- 
creasing the odds of impotence and urinary leakage. Present recommendations have shifted to education and informed participation in screenings rather than blanket screenings (Gattalari \& Ward, 2005). There is a need for targeted and culturally appropriate education that conveys a balanced perspective of the benefits and risks of screening (Taylor et al., 2006).

\section{Methods}

\section{Research Design}

This quasi-experimental, exploratory study used a pre-test/post-test design for men attending community education and screening events. The PROCASE, a 10-item, truefalse written test, was selected to measure knowledge of benefits and risks of prostate cancer screening. The PROCASE was also administered to a comparison group who did not attend these events. Demographic, personal health and family health history questions and attitudes about PC and screening were administered to both groups. Education stressing informed decision-making for PC was provided to attendees by a health educator using a scripted slide presentation, written handouts, and a question and answer session. The post-test was administered immediately after the information session.

The mean total correct knowledge scores for attendees were compared to mean total correct knowledge scores for non-attendees with student $t$-tests. Knowledge acquisition was measured by comparing attendee pre- and post-test scores using paired $t$-tests. Chi-square tests were employed to examine relationships between categorical variables, such as race and ever having had a PSA test, and attendance or nonattendance. Logistic regression was used to create a model predicting attendance at community education and screening events. Covariates included in the model were variables with significant differences based on attendance determined in the earlier analyses.

\section{Procedures}

This project emerged from an alliance between a multidisciplinary team of university researchers, staff from two public health departments and a community health center, as well as local prostate cancer survivors. Applying principles of communitybased participatory research, members of the alliance articulated the purpose, developed research questions and study protocol, and selected research instruments (Minkler \& Wallerstein, 2003). Members of the research alliance selected educational materials that stressed informed decision-making, rather than blanket recommendation for screening. Researchers chose materials culturally appropriate for African-American and white men, written in simple, non-technical language. They refined the proposal, reviewed and consulted on the education protocol, acquired instruments to measure knowledge, developed additional survey items, and secured IRB approval to conduct the study. 


\section{Research Questions}

Three research questions were proposed:

1. Are men who attend community PC education and screening events more knowledgeable of PC than non-attendees?

2. Do attitudes and desire for information about PC differ among men who attend community PC education and screening events vs. non-attendees?

3. What factors predict attendance at community PC education and screening events?

\section{Sampling}

Sampling was self-selective and consisted of 340 men who attended 24 education and screening events held in 19 locations over a nine-month period. Events were advertised in local newspapers, church announcements, and flyers placed in barbershops, local post offices, and grocery stores. They were promoted as educational events with free PSA screening available. A non-random, purposive sample of 87 men residing in the same communities who had not participated in the events was recruited from food pantries, a senior center, a mall, and several fast-food establishments. These locations were suggested by members of the Prostate Cancer Alliance to enlist men for this parallel sample.

\section{Instruments}

The PROCASE Knowledge Test, a 10-item true-false instrument, was used to measure knowledge about risks and benefits of PC screening. The instrument developed was based on items used in previous studies assessing PC knowledge and on expert opinion, followed by review of items for clarity and ease of reading by focus groups of appropriate men. Psychometric testing followed initial item development as part of the 2002 Prostate Cancer Screening (PROCASE) Study, which was a randomized study of 875 male, military-veteran patients receiving healthcare services at four Veterans Affairs centers located in the Midwest (Radosevich et al., 2004). Consensus of 29 clinical experts established criterion validity. A Kuder Richardson-20 $=0.68$ established reliability of the tool. Although the tool demonstrated moderate reliability, the discrimination index average of 0.2 was not an extremely rigorous index score.

\section{Results}

\section{Response Rate}

The study protocol was approved by a University Institutional Review Board and the boards of the two local health departments involved in the project. A written ex- 
planation of the study was provided on the questionnaire and repeated by the health educator at all education and screening events. Participating in the education event was a condition for receiving the free PSA test, although completing pre- and post-tests was not. Twelve men attending education and screening events declined the pre-test, and an additional 67 men did not take the post-test. All men attending the education and screening events requested a PSA.

\section{Description of Participants}

A summary of the demographic characteristics of participants is presented in Table 2. Men who attended community screening and education events were likely to be college educated $\left(46.9 \%\right.$ vs. $\left.29.9 \%, \chi^{2}=14.5, p=.001\right)$, currently married $(62.9 \%$ vs. $\left.42.5 \%, \chi^{2}=15.94, p<.001\right)$, and white $\left(61.7 \%\right.$, vs. $\left.30.6 \%, \chi^{2}=26.54, p<.001\right)$. In this sample non-white men were almost exclusively African-American (98\%).

\section{Correlates of Prostate Cancer Screening Behavior}

All attendees and non-attendees were queried for PC family history, screening history, access to healthcare, and attitudes about PC. Results are summarized in Table 3.

Differences in family history of PC between men screened and men not screened were not significant, nor were there family history differences reported by race.

Table 2

Summary and Comparison of Demographic Characteristics of Sample

\begin{tabular}{|c|c|c|c|c|}
\hline Characteristic & Screened $(\%)$ & Unscreened (\%) & $X^{2}$ & $p$ \\
\hline \multicolumn{5}{|l|}{ Education } \\
\hline No College & $180(53.1)$ & $61(70.1)$ & - & - \\
\hline Some College & $159(46.9)$ & $26(29.9)$ & 8.16 & .004 \\
\hline \multicolumn{5}{|l|}{ Race } \\
\hline White & $209(61.7)$ & $26(30.6)$ & - & - \\
\hline Non-White ${ }^{\mathrm{a}}$ & $130(38.3)$ & $59(69.4)$ & 26.54 & $<.001$ \\
\hline \multicolumn{5}{|l|}{ Age Range } \\
\hline$<50$ & $108(32)$ & $29(34.5)$ & - & - \\
\hline $50-64$ & $148(43.8)$ & $31(36.9)$ & - & - \\
\hline $65-79$ & $71(21)$ & $18(21.4)$ & - & - \\
\hline$>80$ & $11 \quad(3.3)$ & $6(7.1)$ & 3.42 & $\mathrm{NS}^{\mathrm{b}}$ \\
\hline \multicolumn{5}{|l|}{ Marital Status } \\
\hline Not married & $125(37.1)$ & $48(56.5)$ & - & - \\
\hline Married & $212(62.9)$ & $37(43.5)$ & 10.54 & .001 \\
\hline
\end{tabular}

Note. ${ }^{\text {a }}$ Non-White participants were $98 \%$ African-American. ${ }^{b}$ Not significant. 
Table 3

History, Access, Attitudes Towards PC

\begin{tabular}{|c|c|c|c|c|}
\hline Characteristic & Screened $(\%)$ & Unscreened $(\%)$ & $X^{2}$ & $p$ \\
\hline \multicolumn{5}{|l|}{ History } \\
\hline Family history of PC & $70(21)$ & $24(28.6)$ & - & - \\
\hline No family history & $264(79)$ & $60(71.4)$ & 2.23 & NS \\
\hline \multicolumn{5}{|l|}{ Access } \\
\hline Has care provider & $91(28.3)$ & $66(75.9)$ & - & - \\
\hline No care provider & $231(71.7)$ & $21(24.1)$ & 65.62 & $<.001$ \\
\hline Has had PSA & $165(49.3)$ & $48(55.2)$ & - & - \\
\hline Never/don't know PSA & $170(50.7)$ & $39(44.8)$ & .97 & NS \\
\hline \multicolumn{5}{|l|}{ Attitudes } \\
\hline Very worried about PC & $38(11.5)$ & $12(14)$ & - & - \\
\hline Not worried/don'tknow & $292(88.5)$ & $74(86)$ & .38 & NS \\
\hline Important know have PC & $307(94.8)$ & $69(79.3)$ & - & - \\
\hline Somewhat/not important & $17 \quad(5.2)$ & $18(20.7)$ & 20.99 & $<.001$ \\
\hline Wants more information & $220(68.3)$ & $45(52.3)$ & - & - \\
\hline Knows enough & $102(31.7)$ & $41(47.7)$ & 7.63 & .006 \\
\hline
\end{tabular}

Men who attended community screening-education events were more likely to be those without a regular provider of healthcare $\left(71.7 \%\right.$ vs. $\left.24.1 \%, \chi^{2}=65.62, p<.001\right)$. White men were less than non-white men to likely report having a regular care provider $\left(69.5 \%\right.$ vs. $\left.52.2 \%, \chi^{2}=12.63, p<.001\right)$. Men who attended screening were no more likely to have ever had a PSA test than men who didn't attend. In addition, non-white men were more likely than white men to report being unsure or never having had a PSA test $\left(57.8 \%\right.$, vs. $\left.42.6 \%, \chi^{2}=9.42, p=.002\right)$.

Very few men $(12 \%)$ reported being very worried about PC, and worry was not associated with attendance at education and screening events. However, attendees were more likely to report wanting more information about PC than non-attendees ( $83 \%$ vs. $\left.71.3 \%, \chi^{2}=7.63, p=.006\right)$, and more non-white men $(70.9 \%)$ than white men $(54.8 \%)$ wanted additional PC information. $\left(\chi^{2}=9.55, p=.002\right)$. In this sample, more white men $(90.6 \%)$ than non-white men $(84.6 \%)$ responded that it is very important to know whether or not they have PC $\left(\chi^{2}=6.34, p=.04\right)$ as were attendees $(94.8 \%$ vs. $79.3 \%$ for non-attendees, $\chi^{2}=24.36, p<.001$ ).

\section{Knowledge of Prostate Cancer}

There were significant differences in baseline knowledge of men attending education and screening events compared to non-attendees, men attending having a slightly higher baseline knowledge $(t=2.14, p=.03)$. There was a small, but significant in- 
crease of knowledge of men from pre-test to post-test. Pre-test and post-test scores were significantly higher for white participants. See Tables 4 and 5.

Table 4

Knowledge of Prostate Cancer by Attendance

\begin{tabular}{lcccccc}
\hline $\begin{array}{l}\text { Mean PROCASE Score } \\
(10 \text { possible })\end{array}$ & Pre-test & $t$-test & $p$ & Post-test & Paired t-test & $p$ \\
\hline Attendees & 7.22 & - & - & 7.63 & $4.05^{\mathrm{a}}$ & $<.001$ \\
Non-attendees & 6.79 & $2.14^{\mathrm{b}}$ & .03 & NA & - & - \\
\hline
\end{tabular}

${ }^{\mathrm{a}}$ Comparison of pre-test and post test scores of attendees using a paired $t$-test. ${ }^{\mathrm{b}}$ Comparison of pretest scores of attendees vs. non-attendees using unpaired $t$-tests.

Table 5

Knowledge of Prostate Cancer by Race

\begin{tabular}{lccccccc}
\hline $\begin{array}{l}\text { Mean PROCASE Score } \\
(10 \text { possible })\end{array}$ & Pre-test & $t$-test & $p$ & Post-test & Paired $t$-test & $p$ \\
\hline White & 7.65 & - & - & 8.25 & - & - \\
Non-White & 6.48 & $6.19^{\mathrm{a}}$ & $<.001$ & 6.74 & $7.22^{\mathrm{b}}$ & $<.001$ \\
\hline
\end{tabular}

${ }^{\mathrm{a}}$ Comparison of pre-test scores by race using an unpaired $t$-test. ${ }^{\mathrm{b}}$ Comparison of post-test scores by race using an unpaired $t$-test.

\section{Model for Community Education and Screening Attendance}

A model predicting attendance at PC education and screening events was fitted using logistic regression. Demographic variables that demonstrated significant differences between attendees and non-attendees (race, marital status, and education) were included in the regression equation. Access to a healthcare provider was included in the model, along with desire for more knowledge about PC, importance of knowing if one has PC, and baseline knowledge scores on the PROCASE. Results are presented in Table 6.

Being white, not having a healthcare provider, desire for more information, and belief that it is important to know whether one has PC all independently predicted attendance at community education and screening events. In this model, marital status, education, and PROCASE scores were not significant predictors of attendance when the effects of other covariates were held constant. 
Table 6

Logistic Regression Analysis Predicting Attendance at Community Education and Screening Events

\begin{tabular}{|c|c|c|c|}
\hline Factor & $\begin{array}{l}\text { Odds Ratio } \\
\text { (Adjusted) }\end{array}$ & $\begin{array}{l}\text { Confidence } \\
\text { Interval }\end{array}$ & $p$ \\
\hline \multicolumn{4}{|l|}{ Race } \\
\hline White & 2.98 & $1.54-5.78$ & .001 \\
\hline Non-white & Contrast & & \\
\hline \multicolumn{4}{|l|}{ Marital Status } \\
\hline Currently married & 1.01 & $.54-1.90$ & .974 \\
\hline Not married & Contrast & & \\
\hline \multicolumn{4}{|l|}{ Education } \\
\hline College & .58 & $.30-1.10$ & .097 \\
\hline No college & Contrast & & \\
\hline \multicolumn{4}{|l|}{ Access to Care } \\
\hline No provider & 8.89 & $4.84-17.02$ & $<.001$ \\
\hline Has provider & Contrast & & \\
\hline \multicolumn{4}{|l|}{ Attitudes } \\
\hline Important know have PC & .22 & $.09-.56$ & .001 \\
\hline Not important to know & Contrast & & \\
\hline Wants more information & .43 & $.23-.81$ & .009 \\
\hline Has enough information & Contrast & & \\
\hline Baseline PROCASE & 1.04 & $.88-1.27$ & .676 \\
\hline Total model & $R^{2}=.26$ & & $<.001$ \\
\hline
\end{tabular}

\section{Limitations of the Study}

Although the logistic regression analysis controlled for demographic differences between the attendees and the non-attendees, there still may be self-selection bias since the participants were not randomly assigned to the attendance groups. Those attending education and screening events self-selected. Non-attendees were recruited from locations frequented by men in the same communities where education and screening events had been offered. This sampling strategy limits the generalizability of the findings to men in this region. Although statistically significant, change in knowledge from pretest to post-test was minimal. This could be attributable to limitations in the test such as internal reliability or sensitivity to change over time. The PROCASE may not have been the most appropriate test for this population, as it was originally developed for veterans with regular access to health care. Only $4.5 \%$ (43) of the participants in the Radosovich et al. (2004) study were non-white. 


\section{Discussion}

Men who attended education and screening events had more formal education and seemingly had more baseline knowledge of the benefits and risks of PC screening. They were also less likely than men in the parallel sample to have access to healthcare, suggesting their baseline knowledge came from a source other than a healthcare provider, such as health literature. It is unclear from this study where these men obtained their baseline PC knowledge, suggesting the need for further research on this important issue.

Moreover, there were differences in attitudes and desire for information between the two groups. Attendees wanted more information about PC and felt it important to know if they had PC, although they did not feel especially worried about having PC. Men with a family history of PC, however, were more likely to be very worried about their risk of PC, suggesting an appropriate level of concern for their elevated risk status. Further evidence of the importance of these two variables as independent predictors of attendance at community education and screening sites was demonstrated in the logistic regression model. When controlling for other variables, desire for information and importance of knowing PC status continued to predict attendance at education and screening events. Findings from the research questions suggest baseline knowledge of PC can serve as a motivator to seek additional PC information and status. Furthermore, these findings suggest that messages about the importance of knowing what one is at risk for may be more effective than messages evoking fear or worry.

Several other studies (Plowden, 2006; Woods et al., 2004) stated that married men are more likely to be screened for prostate cancer, which suggests wives are involved in the discussion or the decision for men to seek PC information and screening. When comparing marital status alone to attendance in this study, married men were more likely to attend, although when controlling for other variables, marital status did not predict attendance. However, further exploration of the role of spouses in the decision to seek information is suggested.

The majority of men who attended the education and screening events declared they were very likely to be screened, even before hearing the educational presentation on the risks and benefits of screening. In fact, none of the men declined testing, even after hearing the presentation, which stressed informed decision-making. This finding validates the concerns of other researchers (Chan et al., 2003) that men may not be attending to the risk-benefits, but rather assume screening is the best course of action. There does, however, remain the consideration that men who attended education and screening events were previously educated about the risks and benefits of screening, as their higher baseline knowledge scores would suggest.

These community-based education and screening events set out to reach men at highest risk for undetected prostate cancer-African-American men and men without access to healthcare. Education and screening events were conducted at 19 locations throughout the county. Although men who attended education and screening events were more likely to be white, $38 \%$ of the men who attended were non-white, a greater percentage than that of minority men in the county overall. 
According to Illinois Behavioral Risk Surveillance System data (2004-2006), onethird of men over 40 in this county report they have never had a PSA test, while 50 percent of those attending education and screening events indicated they had never had a PSA. Men attending education and screening events were also more likely than nonattendees to report they did not have a personal healthcare provider, yet this was more likely the case for white men than non-white men. These findings suggest the project was partially successful in reaching targeted populations, especially white men without access to care. More intensely focused and expanded efforts may be needed to attract additional African-American men in this county to future education and screening opportunities.

\section{Conclusions and Implications for Future Research}

The reasons for higher incidence and mortality rates from PC in African-American men are complex. Although screening can detect PC in earlier, potentially more treatable stages, there remains controversy over whether or not screening extends or improves quality of life. Until the issue of routine screening is resolved, the best strategy is to offer information about the benefits and risks of screening and to provide access to screening and treatment for men without access to healthcare (Chan et al., 2003; Plowden, 2006; Woods et al., 2004). Unanswered questions demanding additional study include: Where do men who do not have a provider of healthcare obtain their information about PC? Does worry over PC discourage men from obtaining additional information and screening? What role do spouses play in the decision to seek more information and screening for PC? and, How can men be urged to seek more information about PC in order to make an informed decision about screening?

\section{References}

Abbott, R. R., Taylor, D. K., \& Barber, K. (1998). A comparison of prostate knowledge of AfricanAmerican and Caucasian men: Changes from prescreening baseline to post-intervention. Cancer Journal of Science America, 4, 175-177.

Agency for Healthcare Research and Quality (AHRQ). (2006). U.S. Preventative Services Task Force. Retrieved February 25, 2007, from http://www.ahrq.gov/clinic/pocketgd.htm

American Cancer Society. (2008). Cancer facts and figures for African-Americans 2007-2008. Atlanta: American Cancer Society.

Burack, R.C., \& Wood, D.P. (1999). Screening for prostate cancer: The challenge of promoting informed decision making in the absence of definitive evidence of effectiveness. Medical Clinics of North America, 83, 1423-1442.

Chan, E. C. Y., Vernon, S. W., O’Donnell, F. T., Ahn, C., Greisinger, A., \& Aga, D. W. (2003). Informed consent for cancer screening with prostate-specific antigen: How well are men getting the message? American Journal of Public Health, 93, 779-791.

Courtenay, W. H. (2000). Behavioral factors associated with disease, injury, and death among men: Evidence and implications for prevention. The Journal of Men's Studies, 9(1), 81-142. 
Cowart, L. W., Brown, B., \& Biro, D. J. (2004). Educating African-American men about prostate cancer: The barbershop program. American Journal of Health Studies, 19(4), 205-213.

Gattellari, M., \& Ward, J. E. (2005). A community-based randomized controlled trial of three different educational resources for men about prostate cancer screening. Patient Education and Counseling, 57, 168-182.

Healthy People 2010. (2000). U. S. Department of Health and Human Services. Bethesda, MD.

Illinois Behavioral Risk Surveillance System Prostate Cancer Screening County 2004-2006. Retrieved February 2, 2008 from http://app.idph.state.il.us/brfss/countydata.asp?selTopicCounty $=$ prostca\&areaCounty $=\_82 \&$ show $=$ freq $\& y r C o u n t y=3 \&$ form $=$ county $\& y r=\&$ area $=\&$ selTopic $=$

Illinois Department of Public Health. Cancer Statistics 2000-2004. Retrieved February 2, 2008 from http://www.idph.state.il.us/cancer/statistics.htm\#C

McCreary, D. R., Gray, R. E., \& Grace, S. L. (2006). Gender differences in cancer mortality risk perceptions and screening behaviours among adults 40-60 years of age. International Journal of Men's Health, 5, 53-63.

Minkler, M., \& Wallerstein, N. (Eds.). (2003). Community-based participatory research for health. San Francisco, CA: Jossey-Bass Publishers.

Nagler, H. M., Gerber, E. W., Homel, P., Wagner, J.R., Norton, J., Lebovitch, S., et al. (2004). Digital rectal examination is barrier to population-based prostate cancer screening. Urology, 65, 1137-1140.

National Cancer Institute. (n.d.). Health information national trends survey. Retrieved March 13, 2007 from http://www.cancer.gov

National Center for Health Statistics. (2004). Health, United States 2004 with chartbook on trends in health of Americans. U.S. Government Printing Office: Hyattsville, MD.

Nivens, A. S., Herman, J., Weinrich, S. P., \& Weinrich, M. C. (2001). Cues to participation in prostate cancer screening: A theory for practice. Oncology Nursing Society, 28, 1449-1456.

Plowden, K. O. (2006). To screen or not to screen: Factors influencing the decision to participate in prostate cancer screening among urban African-American men. Urologic Nursing, 26, 477-482.

Radosevich, D. M., Partom. M. R., Nugent, S., Nelson, D., Flood, A. B., Holtzman, J., et al. (2004). Measuring patient knowledge of the risks and benefits of prostate cancer screening. Patient Education and Counseling, 54, 143-152.

Reagan, L. J. (1997). Engendering the dread disease: Women, men and cancer. American Journal of Public Health, 87, 1779-1787.

Smith, R. A., Cokkinides, V., \& Eyre, H. J. (2006). American Cancer Society guidelines for the early detection of cancer, 2006. CA: A Cancer Journal for Clinicians, 56, 11-25.

Steele, C. B., Miller, D. S., Maylahn, C., Uhler, R. J., \& Baker, C. T. (2000). Knowledge, attitudes, and screening practices among older men regarding prostate cancer. American Journal of Public Health, 90, 1595-1600.

Taylor, K. L., Jackson, L. D., Turner, R. O., Johnson, L., Schwartz, M. D., Kerner, J. F., et al. (2006). Educating African-American men about the prostate cancer screening dilemma: A randomized intervention. Cancer Epidemiology Biomarkers Prevention, 15, 2179-2188.

U.S. Census. (2005). Population estimates. Retrieved February 2, 2008, from http://www.census.gov

Weinrich, S. P., Boyd, M. D., Bradford, D., Mossa, M. S.,\& Weinrich, M. (1998). Recruitment of African-Americans into prostate cancer screening. Cancer Practice, 6, 23-30.

Weinrich, S., Holdford, D., Boyd, M., Creanga, D., Cover, K., Johnson, A., Frank-Stromborg, M., \& Weinrich, M. (1998). Prostate cancer education in African American churches. Public Health Nursing, 15, 188-195.

Woods, V. D., Montgomery, S. B., Belliard, J. C., Ramírez-Johnson, J., \& Wilson, C. M. (2004). Culture, African-American men, and prostate cancer: What is reality? Cancer Control, 11, 388-396. 
Reproduced with permission of the copyright owner. Further reproduction prohibited without permission. 